\title{
MULTI-STAGE DISCRETE PROGRAMMING OF THE PRODUCTION LINE
}

The paper highlights the problem of multi-stage optimization consisting in determining a timescale of realization orders of objects. Each production stage requires solving a multi-stage discrete programming problem. Production is optimized from the given state of the line to the state in which the line capacity does not allow any further manufacturing. Tools to be replaced in production aggregates are determined by means of heuristic algorithms. Optimization of the manufacturing line is brought to the discrete linear model within each stage.

Keywords: Multi-stage optimization, production optimization, heuristic algorithm, discrete model.

\section{Introduction}

In recent years there has been a constant progress in development of discrete models. These models support solving complex manufacturing tasks. The outstanding progress has been made due to a variety of more powerful and more flexible methods. There are features that are common to all discrete choice models but the constant progress concerning the area of simulation forms new background for the decision maker who has a choice set at his disposal in the form of options. A variety of choice situations can be represented in a more realistic way letting us solve problems which were previously unapproachable. Discrete choice models are to integrate many aspects of the synthetic environment e.g. a choice over products to realize. Discrete simulation is a technique where the simulation is advanced from event time to event time rather than using a continuously advancing time clock as in continuous simulation [1].

A manufacturing system itself can be regarded as a collaborative network of autonomous manufacturing resources in which the responsibility of decision making is also decentralized into individual entities. Therefore, problem complexity of resource management has been drastically increased. Reducing problem complexity by means of iterative decomposition of problems remains a priority [2].

By means of a concrete example, it is possible to apply the method of multiple criteria integer linear programming method in dealing with the problem of determining an optimal optimization [3].

The problem of determining the optimal production plan for a certain period of time can be dealt with efficaciously by the method of multiple criteria programming. The most important problems during planning production is the selection of optimization criteria, the setting of the problem of determining an optimal production plan, the setting of the model of multiple criteria programming in finding a solution to a given problem, the revised surrogate trade-off method, generalized multi-criteria model for solving production planning problem and problem of choosing technological variants in the specific manufacturing industry [4].

There are a lot of dynamic disturbances in manufacturing processes. It is important to identify the discrepancy between planned and actual activities in real-time and also to provide corrective measures by studying the dynamic behavior of complex manufacturing systems. The system responses differently to arrival patterns for customer orders and the existence of various types of real-time events related to customer orders and machine failures. The nearoptimal values of control variables, which improve the manufacturing process, should be verified in practice. The results of extensive numerical investigation must be statistically examined by using analysis of variance [5].

Most engineering optimization algorithms are based on numerical linear and nonlinear programming methods that require substantial gradient information and usually seek to improve the solution in the neighborhood of a starting point. These algorithms, however, reveal a limited approach to complicated real-world optimization problems. If there is more than one local optimum in the problem, the result may depend on the selection of an initial point, and the obtained optimal solution may not necessarily be the global optimum [6].

Knowledge reduction is one of the key issues in real formal concept analysis. A proposed corresponding heuristic algorithm requires numerical experiments to assess its efficiency [7].

\footnotetext{
* Robert Bucki, Franciszek Marecki

Institute of Management and Information Technology in Bielsko-Biala, Poland, E-mail: rbucki@wsi.edu.pl
} 
Mathematical modeling of highly complex manufacturing systems imitating real production systems precedes building the software which helps the operator of the system determine a product to be realized. For simplicity needs modeling is carried out in the proposed synthetic manufacturing environment. Introducing extended specification details leading to creating the proper functional model of the potential manufacturing system allows us to adjust the modeled system to the required configuration of production stands resulting from customers' demand e.g. the number of stands in the manufacturing line, implemented tools in each production stand and a sequence of passing ordered elements to be realized in other available manufacturing plants of identical production possibilities. Orders are accepted distinguishing customers and their demands. Heuristic algorithms choose the production plant and subsequently, orders which are to be realized to meet the stated criterion. The operating principle forms the basis for creating the simulator of the modeled manufacturing system. However, there are production strategies which decide about the moment of beginning realization of the order [8].

The problem of mathematical modeling of the complex system of identical parallel manufacturing plants requires determining the sequence of order elements which are to be realized. The satisfactory solution to this kind of problem must be sought for on condition it meets the stated criteria of production maximization and minimal tool replacement time associated by the necessary bounds The heuristic algorithms for choosing the order for realization as well as algorithms for determining the most acceptable manufacturing plant are responsible for control of each specific information system. Equations of state illustrate the flow of ordered elements through workstations and buffer stores. Tools in each station of the manufacturing system can be represented by e.g. a drill, a metal cutting saw, a profile grinder, a cutter, a polisher, etc. Some of the tools cannot be regenerated and have to be replaced with a new tool of the same parameters while others are subject to the regeneration procedure. However, the regeneration process can be repeated a limited number of times only. There are also buffer stores placed between production stations in each manufacturing sub-subsystem of a bigger system. An information approach to the problem of modeling based on extensive specification details which form the right basis for the subsequent model creation leads to the programming stage during which the model will be shown in the form of the simulator. This should be later verified by implementation to analyze the more and more complex manufacturing tasks. The simulator illustrating functioning of a real production system in the synthetic environment is to be created. Then more practical cases can be analyzed before the real production process is started [9 and 10].

Manufacturing companies are experiencing unprecedented technological change. Some of them remain effective and competitive. Others cannot manage the risk associated with change and the need for innovation and their activities are to be brought to a standstill or even stopped. Business objectives consist in increasing the production output at the lowest possible costs which can be achieved by simulating a production process and all activities within it. A flexible and adaptable approach to suit production activities must be provided by harnessing all detectable limitations in accordance with defined criteria. By defining a plan in terms of the products to be delivered, the creation, quality and appropriateness of those products can be managed and controlled more easily. Once the decisions have been made by means of the simulation process this process will be the normal starting point for producing the plan. An order realizing process consists in identifying the elements of the order, their quality and the way of managing manufacturing. All procedures must be understood and the logistics of creation secured. First of all, we ought to determine the expected effects of the order realization activities. The production system must contain a complete and correct specification of both the final products and also the main intermediate ones. Planning a production process we need to ensure that all charge material deliveries are made in time not to lead to risk situations. Quality control is another issue important to manufacture products of the required quality. All dependencies between the products and the manufacturing system are to be identified long before the production is started. As products are realized in stages, the exact terms cannot be exceeded [11]. The method of multi-stage programming of the production line is shown in detail in [12]. The optimization approach emphasized in it takes into account heuristic algorithms to control the manufacturing process as well as the manufacturing criteria to be met.

\section{Problem formulation}

Production lines are used in many companies as the basic organizational structure of the production system. These lines consist of sequential aggregates which perform operations on manufactured objects. Each aggregate is equipped with a few types of tools which are used during manufacturing objects of a certain type. The sequence of tools in the subsequent aggregates of the production line forms the route of the object. The production line allows manufacturing various products with the use of different technological routes.

Tools in aggregates get worn out throughout the course of manufacturing and must be replaced with new ones (or regenerated ones). If there are certain worn out tools in the aggregate, then this aggregate can carry out operations only on an object for which its tools are still active. This technological line can manufacture only these objects for which there are routes whose capacity allows production. Moreover, the number of certain type objects which can be manufactured with the use of the determined route depends on the maximal wear of the adequate tool in one of the aggregates.

The problem of multi-stage discrete programming of the production line consists in determining a timescale of realization orders of objects which are manufacturing plans in subsequent stages. The number of objects of each type which are to be manufactured is given (in the unknown number of stages). The state of the production line determined by the wear of tools of each aggregate is given as well before each subsequent stage. 
Each stage requires solving a multi-stage discrete programming problem. The effect of such a solution delivers numbers of objects of each type which are to be manufactured. After completing the production stage the state shows no available routes in the production line. This results in the need for replacing tools with new ones (or regenerated ones).

The above analysis leads to the conclusion that worn out tools are to be replaced with new ones (or regenerated ones) in a certain aggregate (or aggregates). The production line is brought to a standstill during the tool replacement process in one aggregate. The replacement times of tools in aggregates differ. Tool replacement is a sequence process. The standstill time of the production line must be minimized. The choice of the aggregate which is subject to a tool replacement process is made by means of heuristic algorithms. For example, tools in an aggregate can be replaced if they are the most worn out (at least one tool is totally worn out). After replacing a certain tool (or tools), some routes become active which results in beginning the next stage of optimization. Sequential replacement of tools in a lot of aggregates increases the flow capacity of the production line; however it increases the standstill time of the line and the total realization time of all orders.

Multi-stage discrete programming of the production line consists in:

1) Production optimization - from the given initial state of the line to the state in which the line capacity does not allow further manufacturing.

2) Replacement of tools in aggregates - chosen by means of heuristic algorithms.

The number of states is not known and should be minimized in order to minimize the realization time of all orders. This number depends on the order amount, the initial state of the line and heuristic replacement rules of tools in aggregates. To determine the aggregates in which tools are to be replaced heuristic algorithms are implemented as the replacement result depends on the current state of orders which are to be realized as well as the current load of the regeneration station.

The formulated problem belongs to the class of non-linear discrete programming. An optimal linear discrete programming problem is solved in each stage; however, transition between subsequent stages is non-linear. This transition consists in the heuristic choice of aggregates in which tools are replaced.

\section{Mathematical model and algorithms}

Optimization of the production line consists in elaborating a mathematical model and dedicated algorithms. Models concern production optimization and replacement of tools in aggregates. Production optimization is brought to a linear programming problem. The aggregate which requires the change of tools after a production stage is determined by means of heuristic algorithms.

\subsection{Mathematical model of the production line}

Let us assume that there is a production line consisting of $M$ aggregates. There are $N$ types of products manufactured in this line. The number $L_{n}, n=1, \ldots, N$ of each product type to be manufactured is given. Manufacturing of the nth product requires installing the $n$th tool, $n=1, \ldots, N$ in each aggregate.

The discussed model assumes that some of the aggregates which manufacture different types of products (e.g. the ith and nth ones) can use the same tool (e.g. the nth one). As a result, it is assumed that the tool allocation matrix $\mathrm{A}$ is given:

$$
A=\left[a_{m, n}\right], m=1, \ldots, M, n=1, \ldots, N
$$

where: $a_{m, n}$ if the $n$th tool is used to realize the $n$th product in the $m$ th aggregate, $a_{m, n}=0$ otherwise.

Routes for each type objects can be determined on the basis of the matrix $A$. So, the route $d_{n}$ for products of the $n$th type, $n=$ $=1, \ldots, N$ is determined as follows:

$d_{n}=\left\langle a_{1, n}, \ldots, a_{m, n}, \ldots, a_{M, n}\right\rangle$

Manufacturing of one piece of the $n$th product in the $m$ th aggregate, $m=1, \ldots, M$ leads to wearing out the $n$th tool which is defined as $z_{m, n}$ (e.g. percents of a new tool).

The state $S$ of the production line is given. It determines the wear of each tool in each aggregate.

The state is defined in the matrix form:

$$
S=\left[s_{m, n}\right], m=1, \ldots, M, n=1, \ldots, N
$$

where: $s_{m, n}$ - the state of the $n$th tool in the $m$ th aggregate.

Let $R_{n}$ be the number of objects of the $n$th type which can be made with the use of the nth route of the production line. This route is determined by tools of the $n$th type in each $m$ th aggregate,

The value is obtained from the formula:

$$
R_{n}=\min \left[\left(1-s_{m, n}\right) / z_{m, n}\right], m=1, \ldots, M
$$

If $R_{n}>0$, then the production line can manufacture objects of the $n$th type. If for each type of the $n$th product, $n=1, \ldots, N$, the condition $R_{n}=0$ is met, then the production line cannot manufacture any object. It means that there exists at least one totally worn out tool in each route $d_{n}, n=1, \ldots, N$.

To resume the production process in the manufacturing line, a certain aggregate (e.g. the jth one) requires replacing its tool. Let us now assume that all tools in the $j$ th aggregate are replaced simultaneously although generally not all of them are totally worn out. After replacing tools in the jth aggregate (e.g. with new ones) 
for new ones, the state of this aggregate takes the value $s_{j, n}=1$ for every $n, n=1, \ldots, N$.

Let us consider the optimization problem of the production line. Its initial state equals $S^{k-1}$ and the state $S^{k}, k=1, \ldots, K$ is calculated when the line is stopped. At the same time in the state $S^{k}, R_{n}=0$ for every $n, n=1, \ldots, N$. The optimization problem in the kth stage consists in determining the maximal sum of manufactured objects $(n=1, \ldots, N)$.

Let $x_{n}^{k}, n=1, \ldots, N, k=1, \ldots, K$ be the number of objects of the $n$th type which can be manufactured in the production line before it is brought to a standstill.

Taking the above into account the optimization criterion takes the form of the sum

$$
Q^{k}=\sum_{n=1}^{N} x_{n}^{k} \rightarrow \max
$$

Moreover, summing concerns only products which are manufactured. The number $N^{k}$ of products manufactured in subsequent stages $k=1, \ldots, K$ decreases because some orders were already realized before the last stage.

At the same time:

$$
\sum_{k=1}^{K} Q^{k}=\sum_{n=1}^{N} L_{n}
$$

The numbers of produced objects are limited by the amount of orders $L_{n}$, so:

$$
\sum_{k=1}^{K} x_{n}^{k} \leq L_{n} \text { for } n=1, \ldots, N
$$

Moreover, in the $k$ th manufacturing stage the state $s_{m, n}^{k-1}$ of the nth tool, $n=1, \ldots, N$ in the $m$ th aggregate, $m=1, \ldots, M$ limits the sum of pieces of manufactured objects. So we can formulate the limitations for each $m$ th aggregate $(m=1, \ldots, M)$

$$
\sum_{n=1}^{N} x_{n} z_{m, n} \leq 1 \text { for } m=1, \ldots, M
$$

\subsection{Optimization algorithms of the manufacturing line}

The formulated discrete model of linear optimization allows us to determine the numbers $x_{1}, \ldots, x_{n}, \ldots, x_{N}$ of products which can be manufactured before the production line is stopped (Fig. 1). These numbers are determined for each stage $k, k=1, \ldots, K$ where $K$ is the stage by which all the orders have been realized. The Integer Programming combinatorial algorithm can be implemented to solve this problem. After stopping the production line, tools must be replaced in a chosen aggregate (e.g. in the jth one). The aggregate is determined by means of the heuristic algorithm. As an example the $j$ th aggregate can be determined on condition its tools are the most used which means the condition below is to be met:

$$
\max _{1 \leq m \leq M} S_{m}^{k}=S_{i}^{k}
$$

At the same time:

$$
S_{m}^{k}=\sum_{n=1}^{N} s_{m, n}^{k}
$$

In a general case an aggregate or aggregates whose tools are to be replaced can be determined by means of another heuristic algorithm.

After the kth stage, $k=1, \ldots, K$ the state of the manufacturing line changes from $S^{k-1}$ to $S^{k}$. If tools in the $j$ th aggregate are replaced, then coordinates of the state $S^{k}$ are determined as follows:

$$
s_{m, n}^{k}=s_{m, n}^{k-1}+\sum_{n=1}^{N} x_{n} z_{j, n} \text { for } m \neq j
$$

and

$$
S_{j, n}^{k}=1 \text { for } n=1, \ldots, N
$$

Moreover, after the $k$ th stage there is a need to correct the bound for the number of manufactured products to the numbers $L_{n}^{k+1}$ where

$$
L_{n}^{k+1}=L_{n}^{k}-x_{n}^{k}
$$

In a general case, the multi-stage discrete programming model lets us determine numbers in a sequential way:

$$
x_{1}^{k}, \ldots, x_{n}^{k}, \ldots, x_{N}^{k}
$$

of products manufactured in the kth stage, $k=1, \ldots, K$.

The following bound is met in the $k$ th stage:

$$
0 \leq x_{n}^{k} \leq L_{n}^{k}
$$

Moreover, after all stages we receive

$$
\sum_{n=1}^{N} x_{n}^{k}=L_{n} \text { for } k=1, \ldots, K
$$

So, during each stage $k, k=1, \ldots, K$ the sum of objects manufactured in each production line is maximized. In this way, the number of stages $K$ is minimized. 


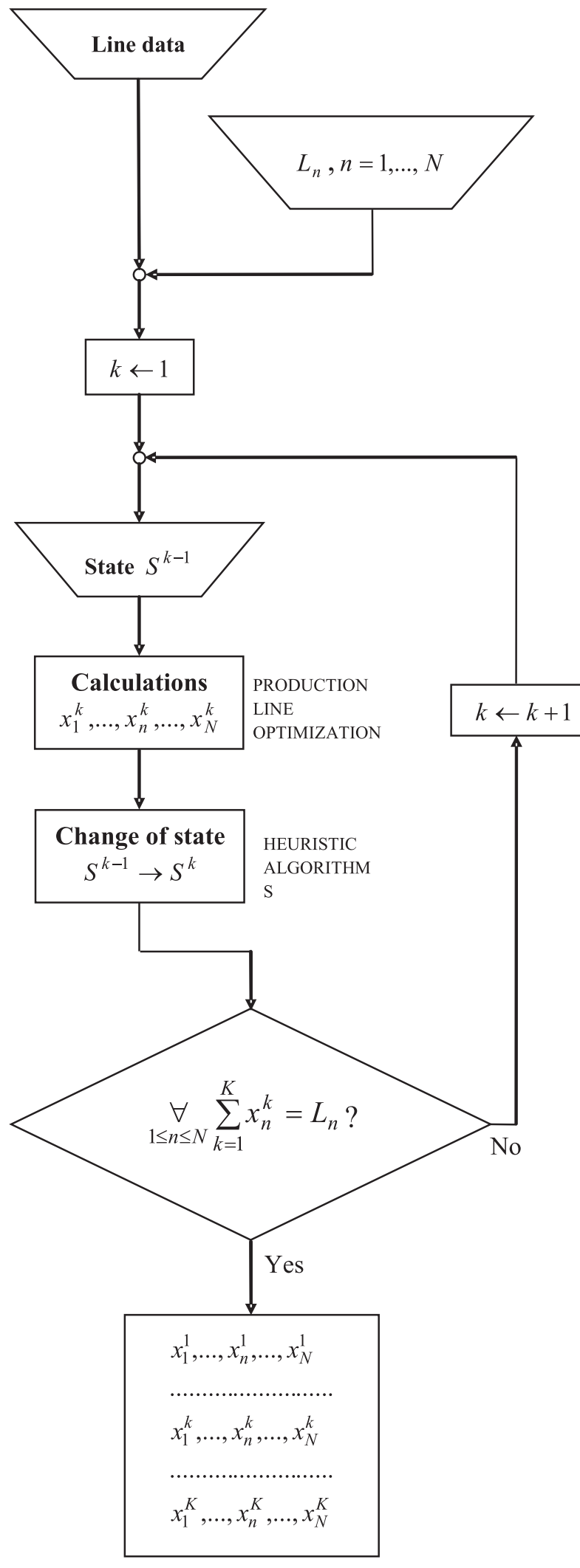

Fig. 1 Multi-stage optimization of the production line

\section{Computer simulators of the production lines}

As the problem of manufacturing line optimization is combinatorial computer simulators are implemented to solve it. Such simulators are based on heuristic algorithms which choose:

- the order to be realized,

- the aggregate whose tools are to replaced.

In some cases orders to be realized and aggregates with tools to be replaced are determined at random. By means of the computer simulator it is possible to generate a big number of allowable alternatives of manufacturing timescales. The currently best solution is chosen from these alternative solutions which is the one realizing all orders in the shortest possible time. Moreover, the histogram of obtained solutions is created when the number of alternative solutions is big (e.g. more than 1000). On the basis of such a histogram it is possible to estimate the probability of determining a better solution during further generating of alternative solutions.

The illustration of production line optimization is described in works [13 and 14] for the production lines of the continuous rolling process. Assemblies of rolls are treated as aggregates. Products of various types are rolled in different routes. Routes are determined by passes of separate assemblies of rolls. Passes in the rolls are worn out during the rolling process. If no more products can be rolled in the rolling mill, the assemblies of rolls of a certain aggregate are exchanged [15]. Generally, equations of state and control of the assembly line are the basis for the process description in the form of equations of state. The equations of state show the state transformation in time under the influence of control [16].

\section{Conclusions}

The problem of production line optimization shown in the article is of a practical value in many uses. The optimization model is deterministic. The wear of tools is given on the basis of linear static characteristics $s_{m, n}=f(x)$ where $x$ is the number of pieces of products. Also, the orders $L_{n}, n=1, \ldots, N$ are given. So, it is the problem of combinatorial optimization. The problem of combinatorial optimization formulated in the paper has been solved with the use of multi-stage programming. The method of discrete multi-stage programming is an original approach to the problem of solving optimization tasks of manufacturing lines. Optimization of the manufacturing line is brought to the discrete linear model within each stage and heuristic replacement of tools after each stage. However, the sum of optimal solutions within each stage does not guarantee global optimization.

The solution to the problem depends on heuristic algorithms of the aggregate choice if it is subject to replacement of tools. Replacement of tools leads to the production line standstill which must be minimized. In a global case, there is a need to minimize the number of tool replacement procedures which in fact is associated with the stages $K$ if tools are replaced sequentially. Moreover, tools can be replaced in a few aggregates simultaneously which justifies the use of heuristic algorithms. The article highlights the 
problem of continuous and discrete processes. State and control equations of the process are introduced in order to define the control in the matrix form.

In a general case, tools can be replaced simultaneously in a few aggregates. The problem of time-scaling of tools replacement in $M$ aggregates by a smaller number of teams $b$ where $b<M$ is NP complete in the sense of its calculation complexity. Additionally, it justifies the use of heuristic algorithms to choose the aggregate in which tools are replaced.

\section{References}

[1] KENNETH, T.: Discrete Choice Methods with Simulation. Cambridge University Press, 2009, p. 408, ISBN 978-0-521-74738-7.

[2] SHIN, M., RYU, K., RYU, T., BAE, S.: A Management Framework for Autonomous and Intelligent Resources in Distributed Manufacturing Systems: A Fractal-based Approach. Advanced Science Letters, vol. 14, No. 1, American Scientific Publishers, 2012, pp. 203-208(6), ISSN 1936-6612.

[3] PERIC, T., BABIC, Z.: Fabric Cutting as the Multiple Criteria Programming Problem. Proc. of AIP Conference, vol. 1089, 2009, pp. 188-199, ISSN 0094-243X.

[4] PERIC, T., BABIC, Z.: Determining Optimal Production Plan by Revised Surrogate Worth Trade-off Method. World Academy of Science, Engineering and Technology 47, 2008, pp. 324-333, ISSN 2010-3778.

[5] GEORGIADIS, P., MICHALOUDIS, C.: Real-time Production Planning and Control System for Job-shop Manufacturing: A System Dynamics Analysis. European J. of Operational Research. vol. 216, No. 1, 2012, pp. 94-104, ISSN 0377-2217.

[6] LEE, K., GEEM, Z. A: New Meta-heuristic Algorithm for Continuous Engineering Optimization: Harmony Search Theory and Practice. Computer Methods in Applied Mechanics and Engineering, vol. 194, No. 36-38, 2005, pp. 3902-3933, ISNN 0045-7825.

[7] LI, J., MEI, C., LV, Y., ZHANG, X.: A Heuristic Knowledge Reduction Algorithm for Real Decision Formal Contexts. Rough Sets and Current Trends in Computing. Lecture Notes in Computer Science. Springer Verlag Berlin Heidelberg, vol. 7413, 2012, pp. 303-312, ISBN 978-3-642-32115-3.

[8] BUCKI, R., SUCHANEK, P., VYMETAL, D.: Information Control of Allocation Tasks in the Synthetic Manufacturing Environment. Intern. J. of Mathematics and Computers in Simulation. North Atlantic University Union. Vol. 6, No. 3, 2012, pp. 324-332, ISSN 1998-0159

[9] BUCKI, R.: Logistic Decision-Making in Modelling and Control of the Complex Production System. Antonyova, A. et al.: Mathematical Modelling in Logistics - Optimum Control. Faculty of Management, University of Presov in Presov, Grafotlac Presov, 2012, pp. 51-62 ISBN 978-80-555-0602-9.

[10] BUCKI, R., MARECKI, F.: Modelling of the Rolling Process. Proc. of the Intern. Conference on Inductive Modelling "ICIM-2002", Lviv, 2002, p. 106-109.

[11] Office of Government Commerce. Managing Successful Projects with Prince2 ${ }^{\mathrm{TM}}$. TSO, 2009, ISBN 9780113310593.

[12] MARECKI, F., BUCKI, R.: Logistyka informatyczna linii produkcyjnej. Proceedings of the International Workshop: Information Logistics. Institute of Management and Information Technology, Bielsko-Biała, 14-15 September 2012. ISBN 978-83-62466-18-4.

[13] BUCKI, R., MARECKI, F.: Digital Simulation of Discrete Processes. Network Integrators Associates. Parkland, Florida, 2006, p. 162, ISBN 978-09-788-6068-4.

[14] BUCKI, R., MARECKI, F.: Modelling and Simulation. Network Integrators Associates. Parkland, Florida, 2005, p. 144, ISBN 97883-891-0594-3.

[15] BUCKI, R.: Minimizing Economic Losses of the Rolling Line. Vedecka konferencia Ekonomia a proces poznavania. Fakulta manazmentu, Presovska univerzita v Presove, 2009, pp. 62-67, ISBN 978-80-555-0141-3.

[16] MARECKI, F.: Foundations of Discrete Systems and Processes Modelling. Information and Telecommunication Systems. Polish Information Processing Society, Bielsko-Biala, 2004, vol. 1, pp. 68-79, ISBN 978-83-607-1632-8. 